Microheaters based on ultrasonic actuation of piezoceramic elements

This article has been downloaded from IOPscience. Please scroll down to see the full text article.

2011 J. Micromech. Microeng. 21085030

(http://iopscience.iop.org/0960-1317/21/8/085030)

View the table of contents for this issue, or go to the journal homepage for more

Download details:

IP Address: 141.211.173.82

The article was downloaded on 06/04/2012 at 16:20

Please note that terms and conditions apply. 


\title{
Microheaters based on ultrasonic actuation of piezoceramic elements
}

\author{
Karthik Visvanathan and Yogesh B Gianchandani \\ Department of Mechanical Engineering, University of Michigan, Ann Arbor, MI, USA \\ E-mail: vkarthik@umich.edu
}

Received 30 March 2011, in final form 2 June 2011

Published 19 July 2011

Online at stacks.iop.org/JMM/21/085030

\begin{abstract}
This paper describes the use of micromachined lead zirconate titanate (PZT) piezoceramic elements for heat generation by ultrasonic energy dissipated within the elements and surrounding media. Simulations based on three-dimensional finite-element models suggest that circular disk-shaped elements provide superior steady-state temperature rise for a given cross-sectional area, volume of the PZT element and drive voltage. Experimental validation is performed using PZT-5A heaters of $3.2 \mathrm{~mm}$ diameter and $0.191 \mathrm{~mm}$ thickness. Single-element heaters and dual-element stacks are evaluated. Although the steady-state temperature generated by these heaters reaches the maximum value at the frequency of maximum electromechanical conductance, the heating effectiveness is maximized at the frequency of maximum electromechanical impedance. Stacked PZT heaters provide 3.5 times the temperature rise and 3 times greater heating effectiveness than single elements. Furthermore, the heaters attain the maximum heating effectiveness when bonded to highly damping and non-conducting substrates. A maximum temperature of $120^{\circ} \mathrm{C}$ is achieved at $160 \mathrm{~mW}$ input power. Experiments are performed using porcine tissue samples to show the feasibility of using PZT heaters in tissue cauterization. A PZT heater probe brands a porcine tissue in 2-3 s with $10 V_{\mathrm{RMS}}$ drive voltage. The interface temperature is $\approx 150{ }^{\circ} \mathrm{C}$.
\end{abstract}

(Some figures in this article are in colour only in the electronic version)

\section{Introduction}

Microheaters have been used in numerous microsystems ranging from gas detectors [1] to mass flow sensors [2], as well as in microfluidic systems for assays [3-5], chemical reactors [6], micro incandescent lamps [7], pressure sensors [8], gas preconcentrators [9] and in microsystems for nanostructure synthesis $[10,11]$. A thin-film gold/titanium microheater on a pyrex bulk substrate has also been reported for microthruster ignition, micro explosive boiling and microsensor applications [12]. The dynamic thermal response characterization of surface micromachined heaters has been described in [13]. Conventionally, microheaters have used joule heating in thin-film resistors that are fabricated from metals or doped semiconductors [14-16]. While this is effective and easily implemented, joule heating can present challenges with parasitic heating of leads, particularly as the magnitude of the resistor decreases. Much effort has been directed at developing heaters with high efficiency and low response time. This has resulted in the investigation of different materials for the heating elements. For example, the moderate heat conductivity of TiN (15 W m $\mathrm{W}^{-1} \mathrm{~K}^{-1}$ ) lowers the conductive losses through the connecting wires, thereby improving efficiency [17]. Microheaters based on a conductive composite of silver microparticles embedded in polydimethylsiloxane (PDMS) have also been reported [18].

Ultrasound has been used widely in the field of medicine for non-invasive heating of biological tissue for hyperthermia and hemostasis [19, 20]. The mechanism relies upon direct absorption of the ultrasound energy within a dissipative medium. At the macroscale, lead zirconate titanate $\left(\mathrm{Pb}(\mathrm{Zr}, \mathrm{Ti}) \mathrm{O}_{3}\right.$ or $\left.\mathrm{PZT}\right)$ is widely used to generate the ultrasound because of its favorable piezoelectric properties and electromechanical coupling. Piezoceramic materials can be incorporated into microsystems by additive processes such as sputtering [21], laser-assisted ablative sputtering [22], sol-gel deposition [23], organometallic chemical vapor deposition [24] and screen printing [25], or by a subtractive process 


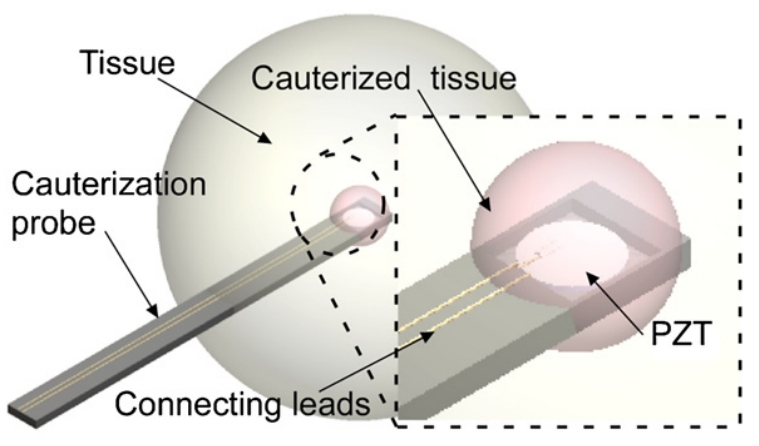

Figure 1. Concept diagram of a PZT-based cauterization tool.

such as ultrasonic micromachining [26]. Microsystems that are based on the ability of PZT to convert electrical energy to mechanical vibrations, and vice versa, include scanning mirror drives [27], micro-optics [28-30], micromixers [31], accelerometer [32], micro-surgery [33, 34] and scanning microscopy probes [35]. This paper explores the possibility of using PZT as a material for microheaters and its possible biomedical applications such as tissue cauterization (figure 1).

Heat generation in free PZT actuators and PZTembedded composite structures is a known phenomenon at the macroscale [36]. The temperature of the piezoelectric (PZT) element increases while converting the electrical energy to mechanical energy. This is mainly due to the dielectric loss in the PZT and the structural damping in the PZT and the surrounding medium. For example, a $(27 \times 10 \times 1) \mathrm{mm}^{3}$ PZT-4S actuator embedded in a $(177.8 \times 165.1 \times 5.08) \mathrm{mm}^{3}$ 50-ply glass epoxy laminate, when excited by $100 V_{\text {rms }}$ at $1000 \mathrm{~Hz}$, has been reported to result in a temperature rise of about $55^{\circ} \mathrm{C}$ above the ambient temperature [36]. Heat generation in multilayered piezoelectric actuators under high frequency of excitation has been studied in [37]. The heat generation is attributed primarily to the ferroelectric hysteresis loss in the stress-free state. An approach to determine the rise in temperature of the PZT element bonded to flat plate structures for sinusoidal voltage actuation has been developed in [38]. The internal heat generation in the PZT element is determined by using a coupled electromechanical model and the temperature field of the PZT actuator is analytically calculated. However, this model does not account for the heat generated in the surrounding medium due to damping of ultrasound waves. The self-heating of PZT actuators has been traditionally considered as a limitation, because the properties of PZT are highly temperature sensitive, and continuous operation at high temperature (close to the Curie temperature) may lead to degradation of the piezoelectric properties.

The temperature of the PZT actuator is dependent on the electric field applied across its electrodes [38]. Hence, for a given voltage, higher temperature can be achieved by reducing the thickness of the PZT element. Thus, microfabricated PZT heaters bear huge potential. The conventional microfabrication techniques for PZT, though capable of achieving a thickness of a few microns, exhibit
Table 1. Properties of the common materials used in fabrication of heaters.

\begin{tabular}{lll}
\hline Material & $\begin{array}{l}\text { Thermal conductivity, } \\
K\left(\mathrm{~W} \mathrm{~m}^{-1} \mathrm{~K}^{-1}\right)\end{array}$ & $\begin{array}{l}\rho c_{p} \times 10^{6} \\
\left(\mathrm{~J} \mathrm{~m}^{-3} \mathrm{~K}\right)\end{array}$ \\
\hline $\mathrm{PZT}-5 \mathrm{~A}$ & $1.1-1.5$ & 2.67 \\
$\mathrm{Cu}$ & 401 & 3.44 \\
$\mathrm{Au}$ & 315 & 2.5 \\
$\mathrm{Si}$ & 150 & 1.6 \\
$\mathrm{Pt}$ & 71.6 & 2.79 \\
\hline
\end{tabular}

degraded material properties and device performance limiting the use of these actuators as microheaters [39]. However, the development of serial and batch-mode bulk ultrasonic micromachining processes has allowed the microfabrication of bulk PZT materials with superior properties, making the proposed device feasible [26].

The PZT-based heaters described here can find applications in thermal microsystems for analytical microsystems and medical devices that benefit from precise heating at moderate temperatures. For example, tissue cauterization requires temperatures less than $200^{\circ} \mathrm{C}$ and the substrates are highly damping and thermally nonconducting (such as polymers and tissues). The high impedance of the PZT element limits the amplitude of the ac current flowing through the system. This can potentially suppress the losses due to parasitic resistances. Furthermore, PZT promises higher thermal efficiency because of its lower thermal conductivity, which results in lower heat loss due to conduction through connecting wires [17]. As shown in table 1, the thermal conductivity of PZT is about two orders of magnitude less than that of metals. The product of density and specific heat capacity for the PZT, which is directly proportional to the time constant of the heaters (for a system with convection boundary condition), is almost the same as that of the metals (table 1). The thermal coefficient of expansion of PZT (which is about $3.8 \times 10^{-6}{ }^{\circ} \mathrm{C}^{-1}$ [40]) is closer to that of single-crystal silicon, silicon nitride, and silicon dioxide than to the metal heaters. Thus, PZT microheaters may cause less thermal stress (due to expansion mismatch) than conventional metal heaters.

This paper ${ }^{1}$ is organized in the following manner. Section 2 describes the mechanism of heat generation in PZT-embedded structures and a 3D finite-element simulation model developed for predicting the temperature rise in the PZT heaters. Section 3 describes the design and fabrication of the heaters used in the experiments. The initial portion of section 4 presents the experimental characterization of the fabricated PZT heaters. The latter portion of section 4 describes the experiments on the cauterization of biological tissue using the proposed PZT heater.

1 Portions of this paper have been published in conference abstract form in [41]. 


\section{Theory and model}

\subsection{Theory}

The heat generation from a piezoelectric material is primarily due to losses within piezoelectric material and attenuation of ultrasound waves in the surrounding medium.

2.1.1. Losses within the piezoelectric material. For a ferroelectric material, there are four sources of losses in the material [42]:

(1) losses due to domain wall motion,

(2) losses due to the presence of point defects in the lattice structure,

(3) microsturctural losses arising from the grain boundaries due to polycrystalline nature of the material and

(4) Ohmic losses (which are relevant mainly for highly conductive materials).

However, in piezoceramic material, the domain wall losses dominate the other three losses. In piezoelectric material, the domain wall losses consist of dielectric, elastic and electromechanical hysteresis losses.

The piezoelectric material is modeled using linear theory of piezoelectricity. In linear piezoelectricity, the following equations govern the behavior of piezoelectric materials [43, 44]:

$$
\begin{gathered}
S_{p}=s_{p q}^{E} T_{q}+d_{k p} E_{k} \\
D_{i}=d_{i q} T_{q}+\varepsilon_{i k}^{T} E_{k}
\end{gathered}
$$

where $T$ is the stress tensor, $S$ is the strain tensor, $E$ is the electric field vector, $D$ is the electric displacement vector, $s_{p q}^{E}$ are the mechanical compliance coefficients, $d_{k p}$ are the piezoelectric coupling coefficients and $\varepsilon_{i k}^{T}$ are the dielectric permittivity coefficients.

In general, there are 36 independent constants for mechanical compliance $\left(s_{p q}^{E}\right), 18$ for piezoelectric coupling $\left(d_{k q}\right)$ and 9 for dielectric permittivity $\left(\varepsilon_{i k}^{T}\right)$. However, due to the orthotropic nature and symmetry in the crystal structure of most PZT materials, the constitutive equations given above can be combined into a single matrix as shown below:

$\left[\begin{array}{c}S_{1} \\ S_{2} \\ S_{3} \\ S_{4} \\ S_{5} \\ S_{6} \\ D_{1} \\ D_{2} \\ D_{3}\end{array}\right]=\left[\begin{array}{ccccccccc}s_{11}^{E} & s_{12}^{E} & s_{13}^{E} & 0 & 0 & 0 & 0 & 0 & d_{31} \\ s_{12}^{E} & s_{11}^{E} & s_{13}^{E} & 0 & 0 & 0 & 0 & 0 & d_{31} \\ s_{13}^{E} & s_{13}^{E} & s_{33}^{E} & 0 & 0 & 0 & 0 & 0 & d_{33} \\ 0 & 0 & 0 & s_{55}^{E} & 0 & 0 & 0 & d_{15} & 0 \\ 0 & 0 & 0 & 0 & s_{55}^{E} & 0 & d_{15} & 0 & 0 \\ 0 & 0 & 0 & 0 & 0 & s_{66}^{E} & 0 & 0 & 0 \\ 0 & 0 & 0 & 0 & d_{15} & 0 & \varepsilon_{11}^{T} & 0 & 0 \\ 0 & 0 & 0 & d_{15} & 0 & 0 & 0 & \varepsilon_{11}^{T} & 0 \\ d_{31} & d_{31} & d_{33} & 0 & 0 & 0 & 0 & 0 & \varepsilon_{33}^{T}\end{array}\right]\left[\begin{array}{c}T_{1} \\ T_{2} \\ T_{3} \\ T_{4} \\ T_{5} \\ T_{6} \\ E_{1} \\ E_{2} \\ E_{3}\end{array}\right]$.

The losses in the piezoelectric material can be modeled by using complex physical constants in these constitutive equations. One assumption in this model is that the losses are small and can be treated as perturbations. The dielectric, elastic and electromechanical coefficients can now be written as [45]

$$
\begin{aligned}
\varepsilon^{T *} & =\varepsilon^{T}(1-\mathrm{j} \tan \delta) \\
s^{E *} & =s^{E}(1-\mathrm{j} \tan \phi) \\
d^{*} & =d(1-\mathrm{j} \tan \theta)
\end{aligned}
$$

where $\delta$ is the phase delay between the electric displacement and the applied electric field under a constant stress, $\phi$ is the phase delay between the strain and the applied stress under a constant electric field and $\theta$ is the phase delay between the electric displacement and the applied stress. The power dissipated within the piezoelectric material is then given by

$$
\begin{gathered}
P=\frac{I_{m} V_{m}}{2} \cos \varphi=\frac{V_{m}^{2}}{2} \operatorname{Re}(A) \\
Q_{p}=\frac{P}{\text { volume(PZT) }}
\end{gathered}
$$

where $V_{m}$ and $I_{m}$ are the magnitude of the actuation voltage and current, respectively, $\varphi$ denotes the phase-angle difference between the current and the voltage, $A$ is the complex admittance of the PZT-embedded structure and $Q_{p}$ is the internal heat generation rate per unit volume in the PZT element. A method for determining the complete set of complex dielectric, elastic and electromechanical coefficients is described in [46].

2.1.2. Losses in the surrounding medium. The ultrasound wave traveling in the surrounding medium is attenuated due to material damping. This energy loss results in heat generation in the surrounding medium. In order to simulate the resulting temperature rise, a steady-state heat-conduction model is used [47]:

$$
\rho_{t} c_{t} \frac{\partial T}{\partial t}=\nabla \cdot k \nabla T+\dot{q}
$$

where $\rho_{t}$ is the density $\left(\mathrm{kg} \mathrm{m}^{-3}\right), c_{t}$ is the specific heat capacity $\left(\mathrm{J} \mathrm{kg}^{-1} \mathrm{~K}^{-1}\right), k$ is the thermal conductivity $\left(\mathrm{W} \mathrm{m}{ }^{-1} \mathrm{~K}^{-1}\right.$ ), $T$ is the temperature $(\mathrm{K})$ and $\dot{q}$ is the heat-generation rate per unit volume due to ultrasound attenuation $\left(\mathrm{W} \mathrm{m}^{-3}\right)$. The heatgeneration rate per unit volume in the surrounding medium is given by

$$
\dot{q}=\alpha \frac{p p *}{\rho_{t} c}
$$

where $\alpha$ is the ultrasound attenuation coefficient, $p$ is the acoustic pressure, $p^{*}$ is the complex conjugate of the pressure and $c$ is the sound wave velocity.

In the absence of a pressure source, the pressure distribution in the surrounding medium due to PZT vibration can be determined by solving the following wave equation:

$$
\frac{1}{\rho_{t} c^{2}} \frac{\partial^{2} p}{\partial t^{2}}+\nabla \cdot\left(-\frac{1}{\rho_{t}}(\nabla p)\right)=0 .
$$

The losses in the medium are modeled using the complex sound velocity as shown below [48]:

$$
\begin{gathered}
k=\frac{\omega}{c}-\mathrm{i} \alpha \\
c_{c}=\frac{\omega}{k} .
\end{gathered}
$$

Here, $c_{c}$ is the complex wave velocity of the medium. 


\subsection{Simulation model}

A 3D multi-physics finite-element model (COMSOL 3.5a Multiphysics $\left.{ }^{\circledR}\right)$ was used to determine the heat generated by the PZT heaters for different geometries, operating frequencies and drive voltages. The simulation was subdivided into three steps. The first step involves modeling the heat generated within the PZT element. The piezo solid module in COMSOL was used to determine the electromechanical admittance of the PZT element, which in turn determined the heat-generation rate within the PZT element. The losses in the PZT element were modeled by using complex values for dielectric, elastic and electromechanical coefficients as shown in equations (4)-(6). The losses in the surrounding medium were modeled by using complex material properties as shown in equation (13). The medium surrounding the PZT element was assumed to be isotropic for wave propagation. Next, the acoustic module was used to determine the pressure waves generated in the surrounding medium by the vibration of the PZT element. The normal acceleration $\left(a_{n}\right)$ of the PZT material due to vibrations is related to the pressure wave generated in the surrounding medium by the following boundary condition at the PZTmedium interface:

$$
n \cdot\left(\frac{1}{\rho_{t}}(\nabla p)\right)=a_{n}
$$

where $n$ is the unit normal vector to the PZT-medium interface. Finally, a generalized heat-transfer module was used to determine the temperature rise in the PZT element and surrounding medium. The heat-generation rate per unit volume in the surrounding medium was calculated using equation (10). The conduction boundary condition was applied at the interface boundaries between the structures. Convection heat flux (to surrounding air) was used at all the external boundaries exposed to the surroundings. The free convection coefficient in air is $6-30 \mathrm{~W} \mathrm{~m}^{-2} \mathrm{~K}^{-1}$ [49]. Hence, the convection coefficient in air was assumed to be $20 \mathrm{~W} \mathrm{~m}^{-2} \mathrm{~K}^{-1}$ in the simulations. The meshing of the $3 \mathrm{D}$ model was performed using the free mesh parameter option in COMSOL. Tetrahedral elements with a maximum element size of $1 \mathrm{~mm}$ were used. The boundaries in the model were meshed using triangular boundary elements. An element growth rate of 2 was used in this simulation model.

\section{Device design and fabrication}

The simulation model described in section 2 is used to determine the most suitable shape for the heater. Simulations were performed to determine the maximum temperature rise in the PZT heaters of different shapes, but with the same volume and cross-sectional area. The heaters were assumed to be bonded to a brass substrate. Shapes such as circle, square and rectangle (length $=2 \times$ width) were considered in the simulations. A voltage of $6 \mathrm{~V}$ (peak-peak amplitude) was applied across the electrodes of the PZT element. Ultrasound attenuation coefficients of 100,170 and $1100 \mathrm{~dB} \mathrm{~m}^{-1} \mathrm{MHz}^{-1}$ (obtained from the experiments described in section 4) were used for the brass substrate, conductive and non-conductive (a)

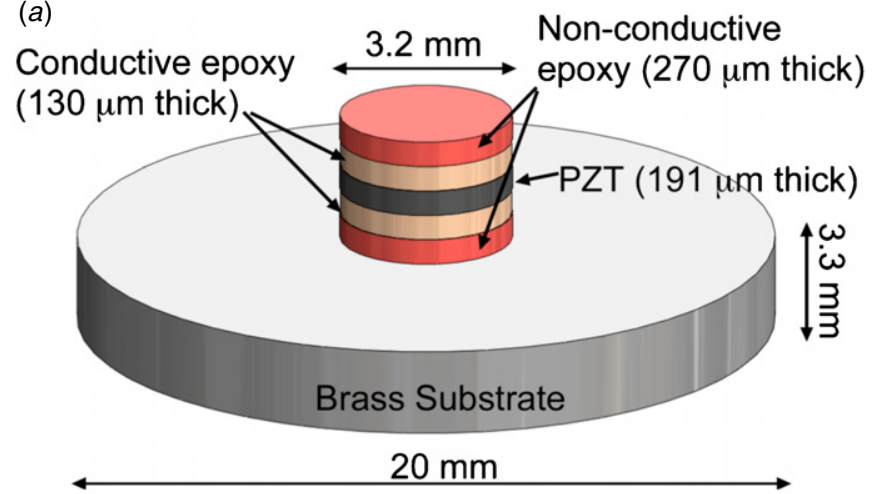

(b)

(c)

PZT

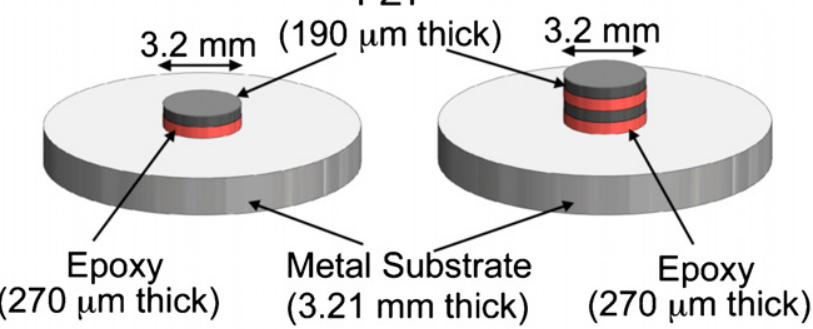

(d)
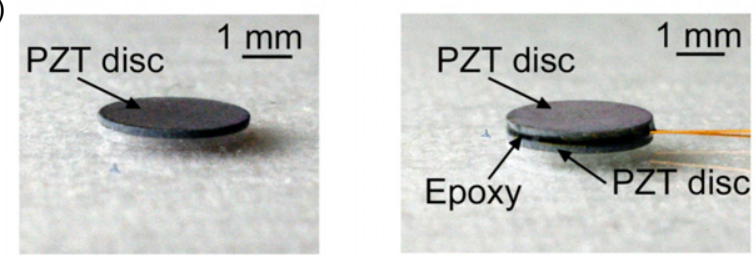

Figure 2. (a) Schematic of the device designed for simulation model validation. Schematic of $(b)$ the unstacked PZT heater and (c) stacked PZT heater. (d) Photograph of the fabricated unstacked and stacked PZT heaters.

epoxies, respectively. The simulations indicated that the circular shape is most suitable for a given volume and crosssectional area. For a given voltage, the circular shape generated $21 \%$ and $39 \%$ more temperature rise as compared to the square and the rectangular shapes, respectively.

This study uses PZT-5A material because it offers higher Curie temperature $\left(\approx 350^{\circ} \mathrm{C}\right)$ than other forms of PZT and consequently allows higher working temperatures. Moreover, the piezoelectric constant $\left(d_{31}\right)$ and relative dielectric constant $(K)$ of PZT-5A show lower temperature sensitivity [43]. Commercially available PZT-5A disks (Piezo Systems Inc., Woburn, MA, USA) of $3.2 \mathrm{~mm}$ diameter and $0.191 \mathrm{~mm}$ thickness were used for experiments.

Figure 2(a) shows the schematic of the device designed for simulation model validation. For the experiments, commercially available PZT heaters of $3.2 \mathrm{~mm}$ diameter and $0.191 \mathrm{~mm}$ thickness were used. The PZT heaters can also be fabricated using a batch-mode ultrasonic machining process [26]. The PZT disk was covered with 130 and $270 \mu \mathrm{m}$ thick layers of conductive and non-conductive epoxies, respectively.

Figures 2(b) and (c) show the schematic and dimensions of the unstacked and stacked structures used for device characterization experiments. For unstacked structures, thin 


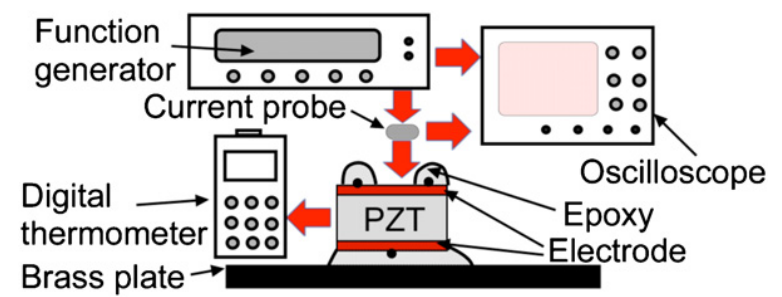

Figure 3. Schematic of the test setup used in the characterization of PZT heaters.

gold bond wires (diameter $\approx 25.4 \mu \mathrm{m}$, length $\approx 10 \mathrm{~cm}$ ) were connected to the PZT disks by conductive epoxy, followed by an insulating layer of non-conductive epoxy. For stacked structures, the PZT disks were bonded in pairs by a thin layer of non-conductive epoxy. Four gold bond wires of $25.4 \mu \mathrm{m}$ diameter (two for each PZT element) were used to provide the electrical connections to the PZT disks as described above. Photographs of the fabricated structures are shown in figure $2(d)$.

\section{Device and operating parameter characterization}

\subsection{Experimental setup}

The experimental setup is illustrated in figure 3 . The heaters were actuated using an HP 33520A function generator. The voltage applied across the electrodes and the current flowing through the heaters were measured using an Agilent DSO6014A oscilloscope and Tektronix CT1 (1 GHz) current probe, respectively. A K-type thermocouple attached to a digital thermometer was used to measure the surface temperature of the PZT heater. The thermocouple was attached to the top of the PZT heater using non-conductive epoxy. The uncertainty in the temperature measurements was $< \pm 0.3{ }^{\circ} \mathrm{C}$, as determined from experimentally measured variations.

\subsection{Simulation model validation}

Simulations and experiments were carried out for circular PZT-5A heaters of $3.2 \mathrm{~mm}$ diameter and $0.191 \mathrm{~mm}$ thickness bonded to $3.3 \mathrm{~mm}$ thick brass substrates (figure $2(a)$ ). The radius of the brass substrate was limited to $10 \mathrm{~cm}$ in order to reduce the complexity of the simulation. Furthermore, due to symmetry only one-quarter of the structure was simulated. Figure $4(a)$ shows the typical meshed geometry used in these simulations. Sinusoidal actuation voltages of peakpeak amplitude 4, 5 and $6 \mathrm{~V}$ were used to determine the ultrasound attenuation in the brass substrate, conductive and non-conductive epoxies. The material properties used in the simulations are listed in table 2.

Experiments were performed to determine the temperatures attained for various actuation frequencies and were compared against the simulation results. Figure 4(b) shows the simulated temperature profile generated for an actuation frequency of $4 \mathrm{MHz}$ and drive voltage of peak-peak amplitude $6 \mathrm{~V}$. The simulation results were
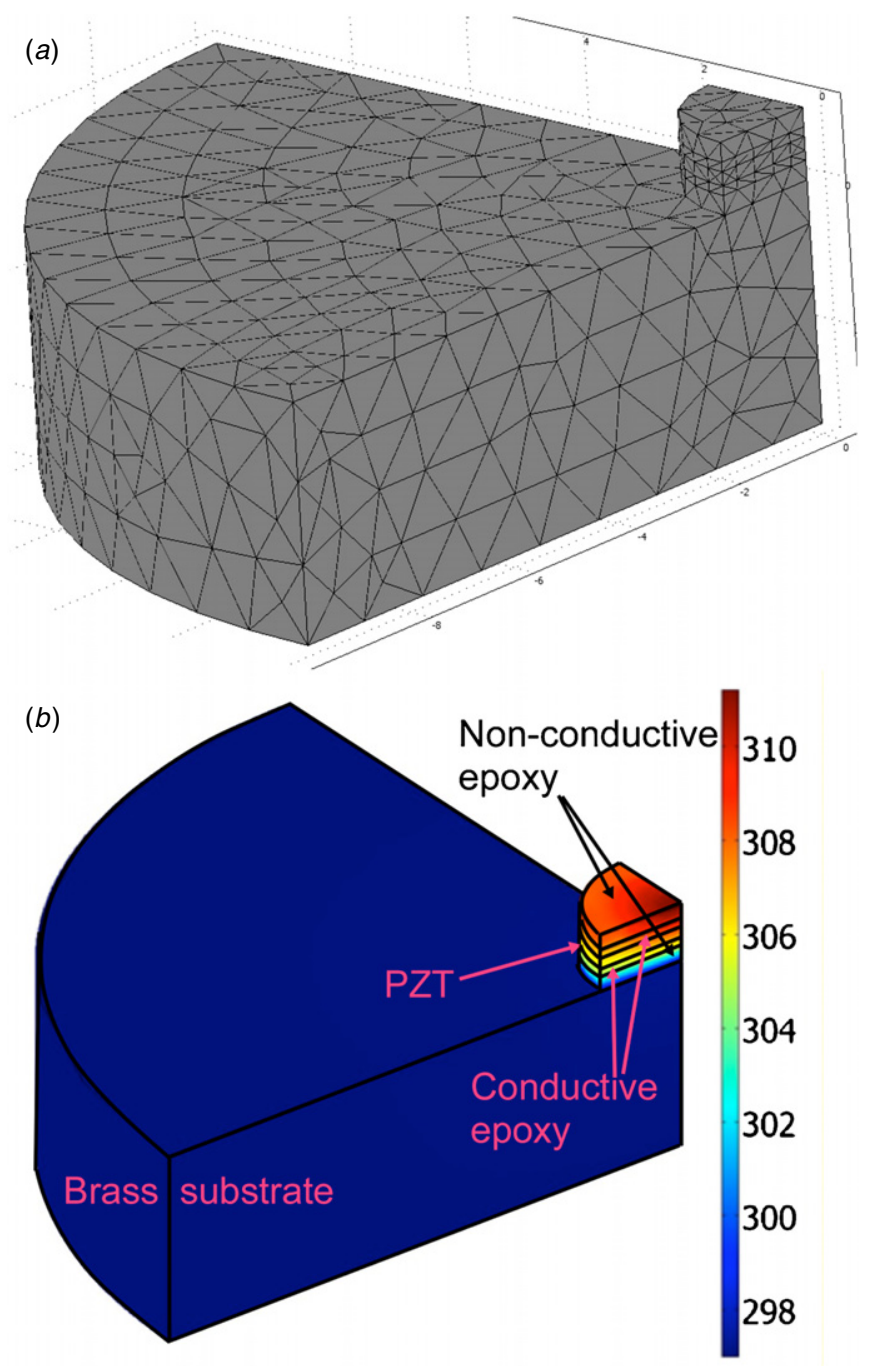

Figure 4. (a) Typical meshed geometry used in the simulations for estimating the temperature rise generated by the PZT heater. $(b)$ The simulated temperature profile generated for a PZT heater bonded to a brass substrate for an actuation frequency of $4 \mathrm{MHz}$ and drive voltage $6 V_{\mathrm{p}-\mathrm{p}}$.

in good agreement with the experimental results for the attenuation values of 100,170 and $1100 \mathrm{~dB} \mathrm{~m}^{-1} \mathrm{MHz}^{-1}$ in the brass, conductive and non-conductive epoxies, respectively (figure 5(a)).

Simulations were also performed to determine the temperature rise for varying input power from the PZT heaters bonded to elytra of a beetle carcass. The simulations were performed using a fitted ultrasound attenuation coefficient of $1000 \mathrm{~dB} \mathrm{~m}^{-1} \mathrm{MHz}^{-1}$ for the elytra of the beetle carcass. The thickness of elytra was assumed to be $200 \mu \mathrm{m}$. The properties of the elytra used in the simulations are listed in table 2. The temperature rise from the PZT heater obtained from simulations was compared with the experiments (figure $5(b)$ ). The simulation and experimental results match within $10 \%$. The difference is believed to be due to nonlinear behavior of the PZT element and the variation of the properties of the PZT element with temperature. 
Table 2. Properties of the materials used in the finite-element simulations.

\begin{tabular}{|c|c|c|c|c|c|c|}
\hline & & Value & Units & & Value & Units \\
\hline \multicolumn{4}{|l|}{ PZT 5A } & \multicolumn{3}{|l|}{ Non-conductive epoxy } \\
\hline \multirow[t]{3}{*}{ Piezoelectric constants } & $d_{31}$ & -175 & $10^{-12} \mathrm{~m} \mathrm{~V}^{-1}$ & Elastic modulus & 0.55 & $10^{10} \mathrm{~N} \mathrm{~m}^{-2}$ \\
\hline & $d_{33}$ & 400 & & Thermal conductivity & 0.5 & 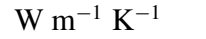 \\
\hline & $d_{15}$ & 590 & & Attenuation & 1100 & $\mathrm{~dB} \mathrm{~m} \mathrm{~m}^{-1} \mathrm{MHz}^{-1}$ \\
\hline \multirow[t]{6}{*}{ Elastic constants } & $c_{11}$ & 6.6 & $10^{10} \mathrm{~N} \mathrm{~m}^{-2}$ & Density & 1096 & $\mathrm{~kg} \mathrm{~m}^{-3}$ \\
\hline & $c_{12}$ & 4 & & Conductive epoxy & & \\
\hline & $c_{13}$ & 4 & & Density & 3000 & $\mathrm{~kg} \mathrm{~m}^{-3}$ \\
\hline & $c_{33}$ & 5.2 & & Elastic modulus & 1.875 & $10^{10} \mathrm{~N} \mathrm{~m}^{-2}$ \\
\hline & $c_{44}$ & 1.2 & & Attenuation & 170 & $\mathrm{~dB} \mathrm{~m}{ }^{-1} \mathrm{MHz}^{-1}$ \\
\hline & $c_{66}$ & 1.25 & & Thermal conductivity & 1.58 & $\mathrm{~W} \mathrm{~m} \mathrm{~m}^{-1} \mathrm{~K}^{-1}$ \\
\hline \multirow[t]{2}{*}{ Dielectric constants } & $\varepsilon_{33} / \varepsilon_{0}$ & 830 & & Brass & & \\
\hline & $\varepsilon_{11} / \varepsilon_{0}$ & 916 & & Density & 8500 & $\mathrm{~kg} \mathrm{~m}^{-3}$ \\
\hline Density & & 7750 & $\mathrm{~kg} \mathrm{~m}^{-3}$ & Attenuation & 100 & $\mathrm{~dB} \mathrm{~m}^{-1} \mathrm{MHz}^{-1}$ \\
\hline Structural damping & & 0.1 & & Elastic modulus & 10.5 & $10^{10} \mathrm{~N} \mathrm{~m}^{-2}$ \\
\hline Coupling loss & & 0.1 & & Thermal conductivity & 115 & $\mathrm{~W} \mathrm{~m} \mathrm{~m}^{-1} \mathrm{~K}^{-1}$ \\
\hline Dielectric loss & & 0.1 & & Beetle's elytra & & \\
\hline Thermal conductivity & & 1.5 & 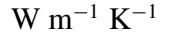 & Attenuation & 1000 & $\mathrm{~dB} \mathrm{~m} \mathrm{~m}^{-1} \mathrm{MHz}^{-1}$ \\
\hline Miscellaneous & & & & Thermal conductivity & 0.4 & $\mathrm{~W} \mathrm{~m} \mathrm{~m}^{-1} \mathrm{~K}^{-1}$ \\
\hline Convection coefficient & & 20 & $\mathrm{~W} \mathrm{~m}^{-2} \mathrm{~K}^{-1}$ & Density & 1000 & $\mathrm{~kg} \mathrm{~m}^{-3}$ \\
\hline Initial temperature & & 300 & $\mathrm{~K}$ & Elastic modulus & 6 & $10^{9} \mathrm{~N} \mathrm{~m}^{-2}$ \\
\hline
\end{tabular}

(a)

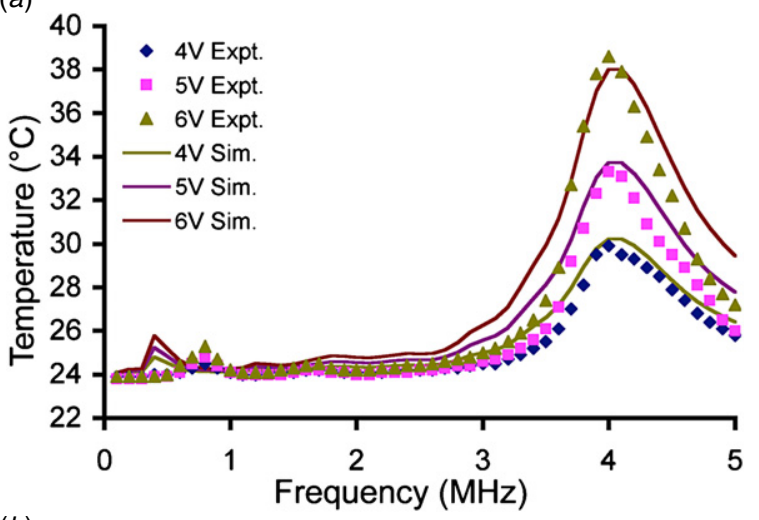

(b)

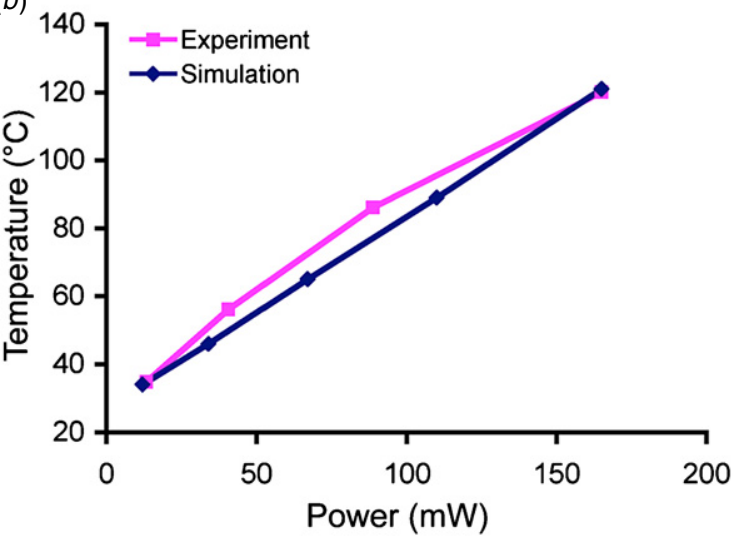

Figure 5. (a) Comparison of the simulated and experimental data for temperature attained for varying applied voltage by PZT elements bonded to a $3.3 \mathrm{~mm}$ thick brass plate. The properties of the material used in the simulation are provided in table 2.

(b) Comparison of the simulated and experimental temperature rise generated by the PZT heater for varying input power when attached to an elytra of a beetle carcass. The simulations were performed with fitted ultrasound attenuation coefficients of 1100, 170, 100 and $1000 \mathrm{~dB} \mathrm{~m}^{-1} \mathrm{MHz}^{-1}$ for the non-conductive epoxy, conductive epoxy, brass substrate and elytra, respectively. The measured heating effectiveness of the heater was $0.93{ }^{\circ} \mathrm{C} \mathrm{mW}^{-1}$ at $85^{\circ} \mathrm{C}$. (a)

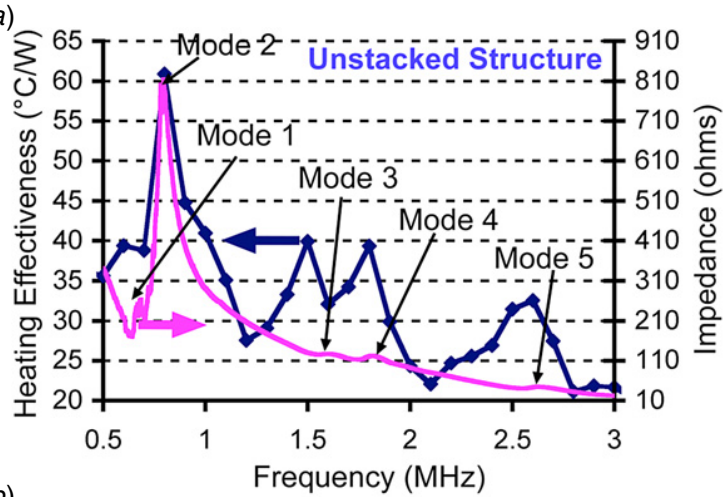

(b)

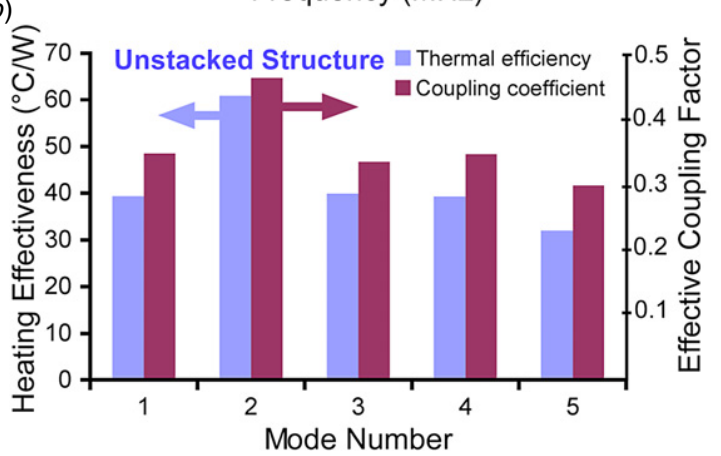

Figure 6. (a) Heating effectiveness and electromechanical impedance of the unstacked PZT heater bonded to a brass substrate as a function of frequency. (b) Heating effectiveness and coupling factor for various mode shapes observed in the unstacked PZT heater bonded to a brass substrate.

\subsection{Operating frequency selection}

The piezoceramic ultrasonic heaters were characterized to determine the most suitable operating frequency. The heaters were bonded to $3.3 \mathrm{~mm}$ thick brass plate using non-conductive epoxy. The heating effectiveness was measured as a function of frequency and compared with the electromechanical impedance of the structure. The heating effectiveness of the heater is defined as the temperature rise per unit watt 

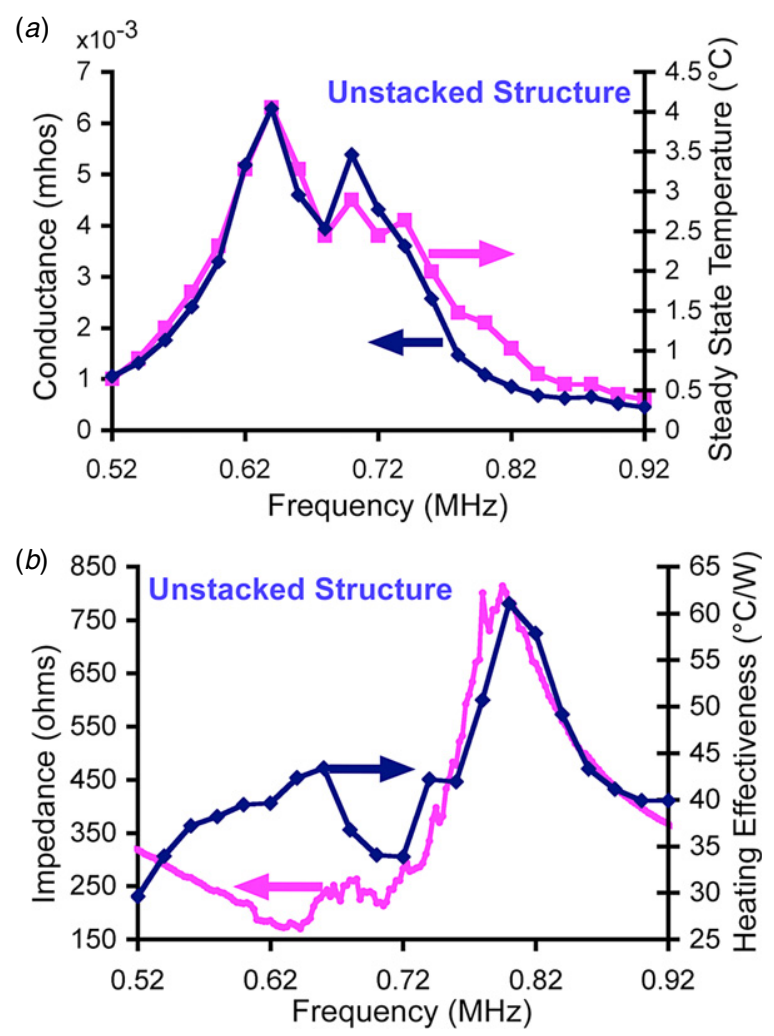

Figure 7. (a) Comparison of temperature attained by PZT and conductance as a function of frequency of excitation for mode 2 on a brass substrate. (b) Comparison of heating effectiveness and electromechanical impedance of the PZT heater as a function of frequency for mode 2 on a brass substrate.

of power consumed. Figure $6(a)$ suggests that the heater attains a local maximum in heating effectiveness around each resonance frequency of the element. The frequencies of modes 1 and 2 were approximately equal to the calculated resonance frequency of the radial mode in the PZT element. Hence, modes 1 and 2 are believed to be radial modes with a small difference in the resonance due to imperfections in the circular shape of the PZT element. Mode 5 is believed to be the higher order radial mode as the frequency is three times the fundamental radial mode frequency. The mode shapes of modes 4 and 5 are not clearly known.

Further analysis was performed to determine the best resonance mode for operation of the PZT heater. Figure $6(b)$ suggests that the heating effectiveness attained in each resonance mode is directly proportional to the effective electromechanical coupling coefficient of the resonance mode. The electromechanical coupling coefficient of a resonance mode is defined as [50]

$$
k_{\text {eff }}=\left(\frac{f_{\mathrm{ar}}^{2}-f_{\mathrm{r}}^{2}}{f_{\mathrm{ar}}^{2}}\right)^{0.5}
$$

where $f_{\mathrm{ar}}$ is the anti-resonance frequency and $f_{\mathrm{r}}$ is the resonance frequency. For the present heater bonded to the brass plate, mode 2 is the optimum. Closer analysis of mode 2 indicates that the heater attains the maximum steadystate temperature for a given voltage at the frequency of maximum electromechanical conductance (figure $7(a)$ ). This is expected, as higher conductance results in higher current amplitude, which, in turn, leads to higher temperature rise. However, the heating effectiveness of the heater is maximum around the frequency of maximum impedance (figure $7(b)$ ). This is believed to be due to decrease in the losses due to parasitic resistances. Hence, there is a compromise in selecting the operating frequency as the maximum steadystate temperature rise per unit voltage occurs at the frequency of maximum conductance, whereas the maximum heating effectiveness occurs at the frequency of maximum impedance.

\subsection{Effect of voltage offset and substrate material}

Experiments indicate that the maximum heating effectiveness occurs at zero dc offset in the drive voltage and that the heating effectiveness decreases with increase in the offset voltage. This is believed to be due to the internal stress generated in the PZT element by the dc offset voltage. This decreases the electromechanical coupling coefficient as discussed in [51]. This, in turn, reduces the heating effectiveness of the heater. Experiments suggest that for an element bonded to a brass plate the heating effectiveness decreases by $28 \%$ for an applied voltage offset of $4 \mathrm{~V}$.

The heating effectiveness of the PZT heater when bonded to different substrates was studied. The heating effectiveness of the heater was higher for substrates with high damping coefficients and lower thermal conductivity, such as biological tissues, and lower for substrates, such as metals. The heaters attained a maximum heating effectiveness of $0.93{ }^{\circ} \mathrm{C} \mathrm{mW}^{-1}$ when bonded to biological tissue (elytra of the beetle). The heating effectiveness was 0.06 and $0.17^{\circ} \mathrm{C} \mathrm{mW}^{-1}$ for brass and glass substrates, respectively.

\subsection{Stacked structure design}

The stacked heater elements (figure 2(c)) were tested in four modes of operation: actuation of bottom element alone, actuation of top element alone, actuation of both elements with the electric field in the same direction and actuation of both elements with the electric field in the opposite direction. Figure 8 shows the comparison of the maximum steadystate temperature and heating effectiveness attained during various modes of operation of the stacked heater. Actuation of the bottom PZT heater alone causes larger temperature rise than unstacked heaters-likely because of the increase in the damping due to the presence of epoxy on both sides of the element. Furthermore, the actuation of the top element alone generates more temperature rise and is more efficient than the lower one-likely because of thermal isolation provided by the lower element. Actuation of both elements within a stack generates the maximum temperature rise and the heating effectiveness is slightly less than that of actuating only the top element. Actuation of both the elements in the stacked structure provides a $3.5 \times$ increase in the steady-state temperature and $3 \times$ increase in the heating effectiveness of the heater when compared to the unstacked heater. The direction of the applied electric field does not have a measurable effect on the temperature rise and the heating effectiveness of the stacked heaters. 


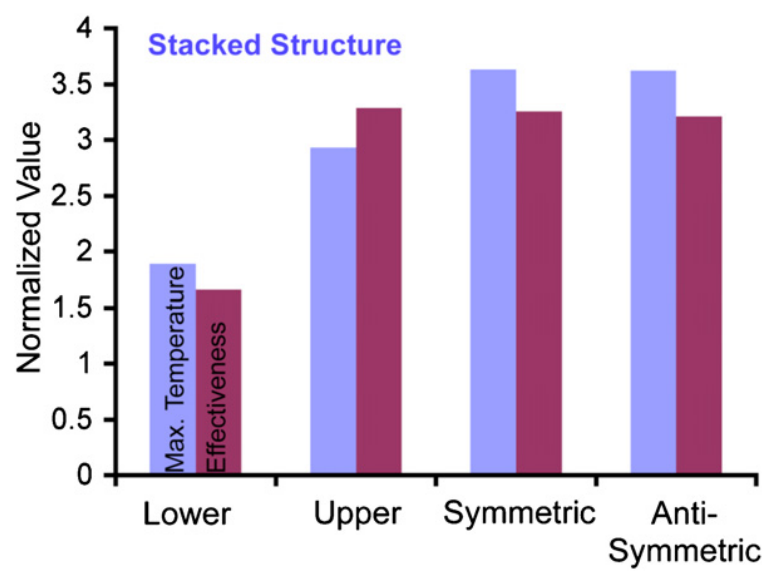

Figure 8. Normalized maximum temperature $\left(T_{\max } / T_{\max -\text { unstacked }}\right)$ and heating effectiveness (eff/eff unstacked $_{\text {) attained by the stacked }}$ PZT heater. The stacked heaters were tested in four modes of operation: actuation of lower element alone, actuation of upper element alone, symmetric actuation of both elements with the electric field in the same direction and anti-symmetric actuation of both elements with the electric field in the opposite direction.

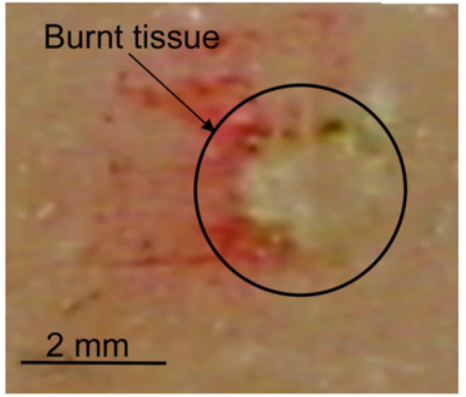

Figure 9. Photograph of the cauterization of porcine tissue using the unstacked PZT heater probe. The heater probe brands the tissue in 2-3 s with $10 \mathrm{~V}_{\mathrm{RMS}}$. The interface temperature is $\approx 150^{\circ} \mathrm{C}$.

\subsection{Biological tissue cauterization experiments}

Experiments were performed using porcine tissue samples to show the feasibility of the microheaters in medical applications. Unstacked circular PZT heaters were used in these experiments. The heaters were bonded to glass substrates by non-conductive epoxy. The voltage output of the function generator was amplified using a Krohn-Hite model 7500 amplifier. The temperature of the PZT heater was measured using a K-type thermocouple bonded to it. The PZT heater probe branded the porcine tissue when a sinusoidal voltage of $10 \mathrm{~V}_{\mathrm{RMS}}$ was applied. The interface temperature rose to $150{ }^{\circ} \mathrm{C}$, which is much greater than the required temperature of $70-100^{\circ} \mathrm{C}$ required for applications such as cauterization [52]. The thermal time constant for the PZT heater bonded to the glass substrate was measured as $11 \mathrm{~s}$. The tissue was branded in 2-3 s after the steady-state temperature was attained. The $3.2 \mathrm{~mm}$ diameter and $0.191 \mathrm{~mm}$ thick heater generated a burn mark of about $2 \mathrm{~mm}$ in diameter as shown in figure 9. This shows the feasibility of using the proposed microheaters for biological tissue cauterization applications.

\section{Conclusions}

There are a number of conclusions that can be drawn based on the findings of this effort. Simulations suggest the circular shape is most suitable to the maximum temperature rise at a given drive voltage for constant cross-sectional area and volume. Circular PZT-5A heaters $(3.2 \mathrm{~mm}$ diameter and $0.191 \mathrm{~mm}$ thickness) were used in experiments. The PZT heaters attained the maximum temperature at the frequency of maximum conductance and maximum heating effectiveness at the frequency of maximum impedance. Furthermore, the heating effectiveness of each resonance mode was proportional to the electromechanical coupling coefficient of that mode. The heating effectiveness of the heaters decreased with an increase in the dc offset of the applied sinusoidal voltage. Furthermore, the heating effectiveness was higher in nonconducting and highly damping substrates. The performance of the stacked PZT structure was also compared with unstacked structure. The stacked structure provided $3.5 \times$ the maximum temperature rise and $3 \times$ the heating effectiveness attained by the unstacked heaters. The direction of the applied electric field did not measurably influence the heating characteristics of the stacked elements. An unstacked heater was used in the cauterization of porcine tissue samples. A sinusoidal input voltage of $10 \mathrm{~V}_{\mathrm{RMS}}$ generated an interface temperature of $150{ }^{\circ} \mathrm{C}$ and branded the porcine tissue. The PZT heaters discussed here bear promise for applications in biomedical devices, where temperatures of the order of $70-200^{\circ} \mathrm{C}$ are required.

\section{Acknowledgments}

KV acknowledges partial support by a fellowship from the Department of Mechanical Engineering, University of Michigan. Partial support for this work was also provided by the Defense Advanced Research Projects Agency Microsystems Technology Office (DARPA MTO).

\section{References}

[1] Najafi N, Wise K D and Schwank J W 1994 A micromachined ultra-thin film gas detector IEEE Trans. Electron Devices 41 1770-7

[2] Moser D, Lenggenhager R and Baltes H 1991 Silicon gas flow sensors using industrial CMOS and bipolar IC technology Sensors Actuators A 27 577-81

[3] Basu A S and Gianchandani Y B 2007 A 128-pixel digitally programmable microfluidic platform for non-contact droplet actuation using Marangoni flows Proc. Int. Conf. on Solid-State Sensors, Actuators, and Microsystems (Transducers) (Lyon, France) pp 771-4

[4] Shin Y S, Cho K, Lim S H, Chung S, Park S J, Chung C, Han D C and Chang J K 2003 PDMS based micro PCR chip with parylene coating J. Micromech. Microeng. 13 768-74

[5] Hennig M and Braun D 2005 Convective polymerase chain reaction around micro immersion heater Appl. Phys. Lett. 87183901

[6] DeMello A J 2006 Control and detection of chemical reactions in microfluidic systems Nature 442 394-402

[7] Mastrangelo C H, Muller R S and Kumar S 1991 Microfabricated incandescent lamps Appl. Opt. 30 868-73 
[8] Mastrangelo C H and Muller R S 1991 Microfabricated thermal absolute pressure sensor with on-chip digital front end processor IEEE J. Solid-State Circuits 26 1998-2007

[9] Yeom J, Field C R, Bae B, Masel R I and Shannon M A 2008 The design, fabrication and characterization of a silicon microheater for an integrated MEMS gas preconcentrator J. Micromech. Microeng. 18125001

[10] Zhang K, Yang Y, Pun E Y B and Shen R 2010 Local and CMOS-compatible synthesis of $\mathrm{CuO}$ nanowires on a suspended microheater on a silicon substrate Nanotechnology 21235602

[11] Engstrøm D S, Rupesinghe N L, Teo K B K, Milne W I and Bøgild P 2011 Vertically aligned CNT growth on a microfabricated silicon heater with integrated temperature control-determination of the activation energy from a continuous thermal gradient J. Micromech. Microeng. 21015004

[12] Zhang K L, Chou S K and Ang S S 2007 Fabrication, modeling and testing of a thin film $\mathrm{Au} / \mathrm{Ti}$ microheater Int. J. Therm. Sci. 46 580-8

[13] Jayaraman B, Bhat N and Pratap R 2009 Thermal characterization of microheaters from the dynamic response J. Micromech. Microeng. 19085006

[14] Chung G S 2004 Fabrication and characterization of micro-heaters with low power consumption using SOI membrane and trench structures Sensors Actuators A $11255-60$

[15] Arata H F, Noji H and Fujita H 2006 Motion control of single F1-ATPase rotary biomolecular motor using microfabricated local heating devices Appl. Phys. Lett. 88083903

[16] Kimura M and Komatsuzaki K 1992 Microheater made of heavily boron doped single crystal silicon beam Technical Digest of the 11th Sensor Symp. pp 169-72

[17] Creemer J F, Van der Vlist W, de Boer C R, Zandbergen H W, Sarro P M, Briand D and de Rooij N F 2005 MEMS hotplates with TiN as a heater material IEEE Sensors Conf. pp 330-3

[18] Liu L, Peng S, Niu X and Wen W 2006 Microheaters fabricated from a conducting composite Appl. Phys. Lett. 89223521

[19] Hynynen K 1997 Review of ultrasound therapy IEEE Ultrasonics Symp. (Toronto, Canada) pp 1305-13

[20] Billard B E, Hynynen K and Roemer R B 1990 Effects of physical parameters on high temperature ultrasound hyperthermia Ultrasound Med. Biol. 16 409-20

[21] Jenkins D F L, Clegg W W, Velu G, Cattan E and Remiens D 1999 The characterization of PZT films of differing orientations for MEMS applications Ferroelectrics 224 259-66

[22] Zhang H, Moilanen H, Usimaki A, Leppavuori S and Rautioaho R 1993 Transformation process of laser ablated PZT thin films J. Electron. Mater. 22 419-22

[23] Cooney T G and Francis L F 1996 Processing of sol-gel derived PZT coatings on non-planar substrates J. Micromech. Microeng. 6 291-300

[24] Peng H C and Desu B S 1994 Metalorganic chemical vapor deposition of ferroelectric $\mathrm{Pb}(\mathrm{Zr}, \mathrm{Ti}) \mathrm{O}_{3}$ thin films $J$. Am. Ceram. Soc. 77 1799-812

[25] Maas R, Koch M, Harris N R, White N M and Evans A G R 1997 Thick-film printing of PZT onto silicon Mater. Lett. 31 109-12

[26] Li T and Gianchandani Y B 2006 A micromachining process for die-scale pattern transfer in ceramics and its application to bulk piezoelectric actuators J. Microelectromech. Syst. 15 605-12

[27] Asai N, Matsuda R, Watanabe M, Takayama H, Yamada S, Mase A, Shikida M, Sato K, Lebedev M and Akedo J 2003 Novel high resolution optical scanner actuated by aerosol deposited PZT films IEEE Int. Conf. on Micro Electro Mechanical Systems (Kyoto, Japan) pp 247-50

[28] Valette S 1995 Micro-optics: a key technology in the race to microsystems J. Micromech. Microeng. 5 74-76

[29] Lin L Y, Lee S S, Pister K S J and Wu M C 1994 Micro-machined three-dimensional micro-optics for integrated free-space optical system IEEE Photonics Technol. Lett. 6 1445-7

[30] Motamedi M E 1994 Micro-opto-electro-mechanical systems Opt. Eng. 33 3505-17

[31] Yang Z, Goto H, Matsumoto M and Maeda R 2000 Active micromixer for microfluidic systems using lead-zirconate titanate (PZT)-generated ultrasonic vibration Electrophoresis 21 116-119

[32] Yu H G, Wolf R, Deng K, Zhou L, Tadigadapa S and McKinstry S T 2001 Fabrication and performance of $\mathrm{d}_{33}$-mode lead-ziconate titanate (PZT) MEMS accelerometers MEMS Components and Applications for Industry, Automobiles, Aerospace, and Communication. Conf. (San Francisco, CA) pp 130-7

[33] Umehara Y and Kurosawa M K 2006 A micro ultrasonic scalpel using hydrothermal PZT thin film 15th IEEE Int. Symp. on the Applications of Ferroelectrics pp 340-3

[34] Ezhilvalavan S, Zhang Z, Loh J and Ying J Y 2006 Microfabrication of PZT force sensors for minimally invasive surgical tools Int. Micro Electro Mechanical Systems Conf. pp 979-84

[35] Cunningham M J, Cheng S T and Clegg W W 1994 A differential interferometer for scanning force microscopy Meas. Sci. Technol. 5 1350-4

[36] Lesieutre G A, Fang L, Koopmann G H, Pai S P and Yoshikawa $S 1996$ Heat generation of a piezoceramic induced-strain actuator embedded in a glass/epoxy composite panel Proc. SPIE 2717 267-75

[37] Zheng J, Takahashi S, Yoshikawa S and Uchino K 1996 Heat generation in multilayer piezoelectric actuators $J$. Am. Ceram. Soc. $793193-8$

[38] Zhou S W and Rogers C A 1995 Heat generation, temperature, and thermal stress of structurally integrated piezo-actuators J. Intell. Mater. Syst. Struct. 6 372-9

[39] Polla D L and Francis L F 1996 Ferroelectric thin films in microelectromechanical systems applications MRS Bull. 21 59-65

[40] Bert C W and Birman V 1998 Effects of stress and electric field on the coefficients of piezoelectric materials: one-dimensional formulation Mech. Res. Commun. 25 165-9

[41] Visvanathan K and Gianchandani Y B 2009 Ultrasonic microheaters using piezo-ceramics for cauterization and other applications IEEE Int. Conf. on Solid-State Sensors, Actuators, and Microsystems (Transducers) (Denver, CO) pp 2421-4

[42] Hardtl K H 1982 Electrical and mechanical losses in ferroelectric ceramics Ceram. Int. 8 121-7

[43] 1987 IEEE Standard on Piezoelectricity

[44] Ikeda T 1996 Fundamentals of Piezoelectricity (New York: Oxford Science)

[45] Uchino K, Zheng J H, Chen Y H, Du X H, Ryu J, Gao Y, Ural S and Priya S 2006 Loss mechanisms and high power piezoelectrics J. Mater. Sci. 41 217-28

[46] Alguero M, Alemany C and Pardo L 2004 Method for obtaining the full set of linear electric, mechanical and electromechanical coefficients and all related losses of a piezoelectric ceramic J. Am. Ceram. Soc. 87 209-15

[47] Meaney P M, Clarke R L, Ter Harr G R and Rivens I H 1998 A 3-D finite element model for computation of temperature profiles and regions of thermal damage during focused ultrasound surgery exposures Ultrasound Med. Biol. 24 1489-99 
[48] Waterman H A 1984 Measurement of the dynamic shear modulus of surface layers J. Colloid Interface Sci. $101377-83$

[49] Kreith F, Manglik R M and Bohn M S 2010 Principle of Heat Transfer (Stratford, CT: Cengage Learning Inc.)

[50] Chen Q and Wang Q M 2005 The effective electromechanical coupling coefficient of piezoelectric thin film resonators Appl. Phys. Lett. 86022904
[51] Cho J, Anderson M, Richards R, Bahr D and Richards C 2005 Optimization of electromechanical coupling for a thin film PZT membrane J. Micromech. Microeng. 15 1797-803

[52] Pritchard W F, Cahen D W, Karanian J W, Hilbert S and Wood B J 2004 Radiofrequency cauterization with biopsy introducer needle J. Vasc. Intervent. Radiol. $15183-7$ 